\title{
Improving postoperative pain control after cesarean delivery with enhanced recovery in patients on buprenorphine therapy
}

\author{
Kevin D. White, MD, MEd', Shirin Azadi, BS', \\ Nadim Bou Zgheib, MD', Brenda L. Mitchell, MD'1
}

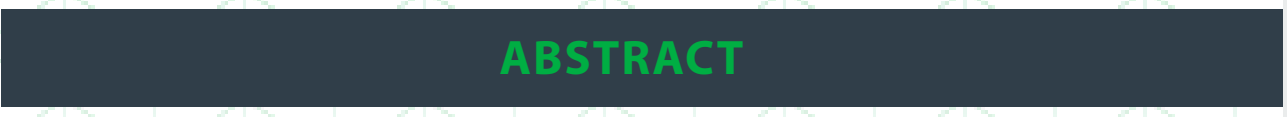

INTRODUCTION: Prescription drug abuse presents a significant challenge to the management of postoperative pain. Pain control amongst the opioid addicted patient can be especially challenging. We aimed to improve pain control after cesarean delivery with enhanced recovery in patients who are on buprenorphine medication-assisted therapy for the treatment of opioid addiction.

Materials and Methods: We conducted a pilot study by implementing a protocol using liposomal bupivacaine injected at the time of cesarean delivery. Patients were then given $500 \mathrm{mg}$ oral acetaminophen every 4 hours, $800 \mathrm{mg}$ oral ibuprofen every 8 hours and $0.3 \mathrm{mg}$ IV buprenorphine every 6 hours as needed. Patients' maintenance dosing of buprenorphine was divided into doses throughout the day. In addition, patients were ambulated 4 hours after surgery and had their catheters removed from their bladder as soon as they could safely ambulate. Eleven patients were prospectively recruited and then compared to a retrospective sample of seventeen patients.

RESULTS: Patients in the treatment group reported $27 \%$ lower pain scores $(p<0.05)$ with $55 \%$ and $100 \%$ achieving a mean pain score 3 and 4 or less, respectively $(p<0.05)$. Patients who were in the treatment group utilized $51 \%$ less breakthrough IV buprenorphine with 45\% declining IV buprenorphine, however these did reach statistical significance. Hospital charges were reduced by $\$ 1,589(p<0.01)$.

DiscusSION: Our enhanced recovery protocol is an effective alternative to traditional pain control and is associated with a significant reduction in both pain scores and use of breakthrough IV buprenorphine as well as lower charges.

\section{KEYWORDS}

buprenorphine, ERAS, pain control, cesarean delivery, liposomal bupivacaine

\section{INTRODUCTION}

Prescription drug abuse creates a significant problem in the United States. With 41.5 overdoses per 100,000, West Virginia has among the highest overdose rates in the country.' Buprenorphine is an approved form of medication-assisted therapy and is recommended for the treatment of addiction in pregnancy. ${ }^{2}$ Pain control for this patient population can be especially challenging, as literature regarding this subject is lacking. Further complicating pain management in this population is the high opioid maintenance dose
Author affiliations are listed at the end of this article.

Correspondence to: Kevin D. White, MD Marshall University Joan C. Edwards School of Medicine whitek@marshall.edu 
Certain protocols have been developed specifically to help patients recover more rapidly after surgery. Enhanced Recovery after Surgery (ERAS) protocols utilize multimodal pain control and local anesthesia combined with early feeding, ambulation and catheter removal to expedite patient recovery. ${ }^{6-12}$ Several studies have shown that ERAS protocols improve patient pain control and increase patient satisfaction while decreasing opioid use..$^{6-12}$ Liposomal bupivacaine is a formulation of the local anesthetic bupivacaine that has been encapsulated by lipids in order to increase the effective duration of the drug by several days. ${ }^{13}$ One study found that using liposomal bupivacaine along with ERAS protocols decreased length of stay following a cesarean delivery without any increase in adverse events. $^{6}$

There is a paucity of data evaluating any manner of pain control after cesarean delivery in patients who struggle with opioid addiction, let alone those on buprenorphine medication-assisted therapy. Data evaluating liposomal bupivacaine is limited to the aforementioned study. We hypothesize that implementation of an ERAS protocol that utilizes liposomal bupivacaine at the time of surgery will improve pain control and decrease the use of IV buprenorphine for breakthrough pain in patients undergoing a cesarean delivery who are in opioid-addiction recovery programs that utilize buprenorphine for medication-assisted therapy.

\section{MATERIALS AND METHODS}

We conducted an ambispective pilot study to evaluate postoperative pain management strategies. This started with a retrospective analysis of the current standard of care. At our institution, patients who undergo scheduled cesarean delivery receive spinal anesthesia consisting of $13 \mathrm{mg}$ bupivacaine, $10 \mathrm{mcg}$ of fentanyl and $0.2 \mathrm{mg}$ morphine. Currently, in addition to the On- ${ }^{\circledR}$ and continuing current home maintenance buprenorphine dose as scheduled, patients receive IV ketorolac $30 \mathrm{mg}$ every 6 hours followed by $10 \mathrm{mg}$ oral ketorolac every 6 hours, as well as $0.3 \mathrm{mg}$ IV buprenorphine every 6 hours as needed. The On- $\mathrm{Q}^{\circledR}$ system utilizes percutaneous catheters that infiltrate the abdominal and pelvic cavities with buprenorphine over a 2 to 3 day period. After surgery, patients are allowed regular diet 1 hour after surgery. The catheter is removed after 12 to 24 hours and patient is allowed to ambulate 12 hours after surgery. The retrospective analysis included patients within given time period that met inclusion criteria.

Next, we prospectively implemented an ERAS protocol previously described in gynecologic oncology literature. ${ }^{13}$ This protocol consisted of the spinal anesthesia, as mentioned above, followed by $266 \mathrm{mg}(20 \mathrm{~mL})$ diluted into an additional $20 \mathrm{~mL}$ of normal saline of liposomal bupivacaine injected subcutaneously along the length of the incision at time of skin closure. Postoperative pain was controlled with $500 \mathrm{mg}$ of oral acetaminophen scheduled every 4 hours, $800 \mathrm{mg}$ of oral ibuprofen every 8 hours and $0.3 \mathrm{mg}$ IV buprenorphine every 6 hours as needed for breakthrough pain. Maintenance buprenorphine dose was then divided into 4 times daily dosing. For example, if a patient takes $16 \mathrm{mg}$ daily of buprenorphine then they would receive $4 \mathrm{mg}$ every 6 hours postoperatively. Patients resumed regular diet 1 hour after surgery. In addition, patients were ambulated after 4 hours and the catheter was removed when the patient could safely ambulate.

We compared the retrospective data, which reflected the standard of care, to the data we collected prospectively following the implementation of the ERAS protocol. For the retrospective analysis, we included all eligible patients over a 1-year span. To be eligible for inclusion in the retrospective group, patients had to be at an age of 18 years or greater, at a gestational age of 34 weeks or greater, and have a non-emergent cesarean delivery at Cabell Huntington Hospital while being treated for opioid addiction with medication-assisted therapy using buprenorphine. For eligibility for inclusion in the prospective group, patients had to meet the same criteria as described for the retrospective group. With informed consent, we recruited a prospective group for the implementation of the ERAS protocol over a 6-month period. Patients were excluded from the study if any one of the above-mentioned criteria were not met. 
We then conducted a retrospective evaluation of medical records for several data points.

These included: patient demographics, medical comorbidities, gravidity, parity, postoperative pain scores, postoperative complications, length of stay (LOS), postoperative care charges, and IV buprenorphine use during hospitalization. Routine patient care also included the collection of urine drug screens. The administration of all postoperative drugs began when the patient left the operative suite, as recorded by the nursing staff.

Mean pain scores served as the primary outcome. On the day of surgery and postoperative days 1 , 2 and 3, the mother-baby nurse recorded pain scores on a Likert scale of 0-10 with 0 representing no pain and 10 representing severe pain. The goal at our institution was for the patient to meet a pain score of 3 or less. Because other institutions use the measure of a pain score of 4 or less, both data points were recorded. We also measured several secondary outcomes, including the amount of IV buprenorphine used, LOS, nausea and/or vomiting that required the use of anti-emetics as well as hospital and pharmacy charges. These charges included the cost of the local anesthetic used intraoperatively as well as the charges for medications used to control pain and nausea and/ or vomiting post operatively. A unique identifier was given to every patient. The master code for these identifiers was securely stored on a password-protected computer. The retrospective pre-implementation data and the prospective postimplementation data was compared using the Fisher Exact test and the Mann Whitney $U$ test. Because data collection was recorded as part of routine postpartum documentation, blinding of patients, providers, and staff was virtually impossible. However, care was taken to ensure that the research staff did not interact with the patient following informed consent, except as medically necessary for routine obstetric and gynecological care. Our study protocol was approved by the Institutional Review Board who found it to be exempt from full review due to the low risk that implementing the protocol posed to the research subjects. Informed consent was also waived for those subjects in the retrospective cohort. The authors of this study do not have any financial disclosures.

\section{RESULTS}

Seventeen patients met inclusion criteria and were included in the MARC cohort retrospective control group. Eleven patients met inclusion criteria for the prospective ERAS group for analysis. Due to the lowrisk nature of the study, none of the eligible patients declined participation in the study. As shown in Table 1, overall mean age was 30.5 years with a range of 23-41 years and a standard deviation of 4.5 years. Mean BMI was $30.9 \mathrm{~kg} / \mathrm{m} 2$ with a range of $20-42 \mathrm{~kg} /$ $\mathrm{m} 2$ and a standard deviation of $6.3 \mathrm{~kg} / \mathrm{m} 2$. The ERAS group had a higher obesity rate $(34.0 \%$ versus $28.4 \%$, $\mathrm{p}<0.01$ ), however the distribution among the various classes of obesity were similar. There were more smokers in the ERAS group 82 versus 71 percent $(p<0.05)$. All patients in both the retrospective and prospective groups were on $8 \mathrm{mg}$ of sublingual buprenorphine twice daily preoperatively. Pregnancy demographics, surgical characteristics and the comorbidities of hypertension and diabetes mellitus were similar between the groups. All urine drug screens were only appropriately positive for buprenorphine.

Overall pain scores were $27 \%$ lower in the ERAS group ( 2.95 versus $4.04, p<0.05$ ), as shown in Table 2. 100 percent of patients in the ERAS group met the pain score goal of 4 or less versus $53 \%$ in the control group $(p<0.05)$. Likewise, $55 \%$ of the ERAS patients versus $18 \%$ of the control group $(p<0.05)$ met the pain score goal of 3 or less (odds Ratio $5.6,95 \% \mathrm{Cl}$ $1.00-31.3$ ). The odds ratio for the pain goal of 4 or less could not be calculated as 100 percent of the ERAS group met this goal.

Similarly, ERAS patients had a lower incidence of days with significant pain, 5 or higher, at 47 versus 64 percent $(p=0.29)$ and lower incidence of days with a severe pain score, 7 or higher, at 0 versus 12 percent $(p=0.70)$, although these did not reach statistical significance. Likewise, ERAS subjects had a lower proportion of those who reported persistent pain of 5 or greater ( 0 versus 18 percent, $p=0.21$ ) and persistent severe pain of 7 or greater ( 0 versus 6 percent, $p=0.61$ ), however these did not reach statistical significance.

As shown in Table 3, overall IV buprenorphine use was $51 \%$ lower in the ERAS group when 
TABLE 1. Demographic and Surgical Characteristics $(n=28)$

\begin{tabular}{|c|c|c|c|}
\hline Characteristic & Control $(n=17)$ & ERAS $(n=11)$ & p Value \\
\hline \multicolumn{4}{|l|}{ Demographic } \\
\hline Age(y) & $17[30.9(29.0-32.9)]$ & $11[28.4(26.6-32.8)]$ & $0.50^{\ddagger}$ \\
\hline BMI & $17[28.4(26.1-30.8)]$ & $11[34.0(30.3-37.7)]$ & $0.01^{\ddagger}$ \\
\hline Normal or overweight & $12(71)$ & $3(27)$ & 0.078 \\
\hline Class I and II obesity & $4(24)$ & $5(45)$ & \\
\hline Morbid Obesity & $1(5)$ & $3(27)$ & \\
\hline Smoking & & & $0.02^{5}$ \\
\hline Never & $5(29)$ & $0(0)$ & \\
\hline Former & $0(0)$ & $2(18)$ & \\
\hline Current & $12(71)$ & $9(82)$ & \\
\hline \multicolumn{4}{|l|}{ Comorbidities } \\
\hline Hypertension & $3(18)$ & $4(36)$ & $0.54^{\S}$ \\
\hline GHTN, Preeclampsia & $2(12)$ & $3(27)$ & \\
\hline CHTN & $1(6)$ & $1(9)$ & \\
\hline Diabetes Mellitus & $0(0)$ & $1(10)$ & $>0.998$ \\
\hline Pre-pregnancy & $0(0)$ & $0(0)$ & \\
\hline Gestational & $0(0)$ & $1(13)$ & \\
\hline \multicolumn{4}{|l|}{ Pregnancy Demographics } \\
\hline Parity & & & $>0.99^{\S}$ \\
\hline Primiparous & $1(6)$ & $1(9)$ & \\
\hline Multiparous & $16(94)$ & $10(91)$ & \\
\hline EGA at time of delivery & $17\left[38^{2 / 7}\left(37^{5 / 7}-39^{0 / 7}\right)\right]$ & $11\left[37^{3 / 7}\left(35^{2 / 7}-39^{4 / 7}\right)\right]$ & $>0.99:$ \\
\hline \multicolumn{4}{|l|}{ Surgical Characteristics } \\
\hline Primary Cesarean Delivery & $3(18)$ & $2(18)$ & $0.68^{8}$ \\
\hline Indication & & & $>0.99 \S$ \\
\hline Repeat & $14(82)$ & $9(82)$ & \\
\hline Malpresentation & $1(6)$ & $1(9)$ & \\
\hline Obstructed Labor & $2(12)$ & $1(9)$ & \\
\hline Anesthesia & & & $>0.99^{\S}$ \\
\hline Spinal & $14(82)$ & $9(82)$ & \\
\hline Epidural & $3(18)$ & $2(18)$ & \\
\hline Tubal Ligation & $6(40)$ & $6(50)$ & $0.44^{5}$ \\
\hline Birth Weight (g) & $17[3116(2858-3373)]$ & $11[2868(2362-3373)]$ & $0.31^{\ddagger}$ \\
\hline $\mathrm{EBL}(\mathrm{mL})$ & $17[641(588-695)]$ & $11[555(483-626)]$ & $0.06^{ \pm}$ \\
\hline 1 minute APGAR 7 or greater & $17(100)$ & $10(91)$ & $0.39^{\S}$ \\
\hline \multicolumn{4}{|l|}{ Data are n [mean $(95 \% \mathrm{CI})]$ or n $(\%)$} \\
\hline \multicolumn{4}{|l|}{ Mann-Whitney U Test } \\
\hline \multicolumn{4}{|l|}{$\S$ Fisher Exact } \\
\hline \multicolumn{4}{|l|}{ GHTN $=$ Gestational hypertension } \\
\hline $\mathrm{CHTN}=$ Chronic hypertension or chroni & pertension with superin & sed preeclampsia & \\
\hline
\end{tabular}

TABLE 2. Primary Outcomes $(n=28)$

\begin{tabular}{|c|c|c|c|c|}
\hline Characteristic & Control (n=17) & ERAS (n=11) & Change & p Value \\
\hline \multicolumn{5}{|c|}{ Markers of Effective Pain Control } \\
\hline \multicolumn{5}{|l|}{ Pain Score } \\
\hline Day 0 & $17[4.37(2.82-5.93)]$ & $11[3.82(1.66-5.98)]$ & $-13 \%$ & $0.66^{\ddagger}$ \\
\hline Day 1 & $17[4.77(3.54-6.00)]$ & $11[2.77(1.47-4.07)]$ & $-42 \%$ & $0.03^{\ddagger}$ \\
\hline Day 2 & $17[4.02(3.02-5.02)]$ & $11[3.46(2.43-4.50)]$ & $-14 \%$ & $0.46^{\ddagger}$ \\
\hline Day 3 & $16[3.03(1.94-4.12)]$ & $8[1.81(0.00-3.63)]$ & $-40 \%$ & $0.23^{\ddagger}$ \\
\hline Mean & $17[4.04(3.31-4.76)]$ & $11[2.95(2.26-3.63)]$ & $-27 \%$ & $0.04^{\ddagger}$ \\
\hline Achieved Pain Goal $(\leq 3)$ & $3(18)$ & $6(55)$ & & $0.05^{\S}$ \\
\hline Achieved Pain Goal $(\leq 4)$ & $9(53)$ & $11(100)$ & & $0.007^{\S}$ \\
\hline \multicolumn{5}{|l|}{ Markers of Ineffective Pain Control } \\
\hline Days with Mean Score > & & & & 0.298 \\
\hline 1 or more days & $8(47)$ & $7(64)$ & & \\
\hline 2 or more days & $6(35)$ & $1(9)$ & & \\
\hline 3 or more days & $1(6)$ & $0(0)$ & & \\
\hline \multicolumn{2}{|c|}{ Days with Mean Score $>7$} & & & $0.70^{5}$ \\
\hline 1 or more days & $2(12)$ & $0(0)$ & & \\
\hline 2 or more days & $1(6)$ & $0(0)$ & & \\
\hline \multicolumn{5}{|c|}{ Persistent pain with mean pain score of: } \\
\hline 5 or greater & $3(18)$ & $0(0)$ & & $0.21^{8}$ \\
\hline 7 or greater & $1(6)$ & $0(0)$ & & $0.61^{5}$ \\
\hline
\end{tabular}


compared to the control group, however, this did not reach statistical significance $(0.53 \mathrm{mg}$ versus $1.08 \mathrm{mg}, p=0.18$ ). Although not statistically significant, patients were more likely to go without IV buprenorphine entirely (45 versus 24 percent, $p=0.22$ ). The range of opioid use in the control group was $0 \mathrm{mg}$ to $4.2 \mathrm{mg}$ with a standard deviation of $1.3 \mathrm{mg}$. The range of opioid use in the ERAS group was $0 \mathrm{mg}$ to $1.8 \mathrm{mg}$ with a standard deviation of $0.7 \mathrm{mg}$. While not statistically significant, patients were less likely to need a breakthrough antiemetic at 9 versus 29 percent $(p=0.21)$. Day 2 discharge rate was higher in the ERAS group at 36 versus 6 percent, but likewise did not reach statistical significance $(p=0.15)$. Pharmacy charges were $\$ 603$ lower $(\$ 1581$ versus $\$ 2184, p<0.01)$. Overall hospital charges were $\$ 1589$ lower ( $\$ 10106$ versus $\$ 11695, p<0.01)$.

None of the patients abandoned the protocol to return to traditional pain control methods. None of the patients in either the retrospective or prospective groups took narcotics aside from buprenorphine. With regard to the liposomal bupivacaine, none of the patients reported allergy, wound infection, injection site reaction or any other complications. Despite early ambulation and early catheter removal, we did not have any adverse events such as urinary retention or falls. All patients completed the study as no patients withdrew consent.

TABLE 3. Secondary Outcomes Patients $(n=28)$

\section{DISCUSSION}

Treating opioid addicted patients represents a special challenge. The goal is to avoid excess narcotics to prevent relapse while still adequately treating postsurgical pain so as to help prevent patients from self-medicating. Our protocol was associated with a $27 \%$ reduction in mean pain scores. Patients in the ERAS group were 5.6 times more likely to reach the pain goal of 3 or less with $100 \%$ of the patients reaching a mean pain score of 4 or less despite all of the control patients using the On- $\mathrm{Q}^{\circledR}$ bupivacaine system. We observed a lower incidence of persistent severe pain in the ERAS, however this did not reach statistical significance.

Our protocol appeared to be associated with dramatic reductions in IV buprenorphine use at 51\%, however this did not reach statistical significance. This statistic was underpowered for this outcome due to the large variability. A secondary MannWhitney test showed to have $80 \%$ power with an alpha of 0.05 to detect an opioid reduction of $51 \%$ was 130 patients per group due to the variability in IV buprenorphine use, which was not feasible for this pilot study. Given the price difference between the On- $\mathrm{Q}^{\circledR}$ system and liposomal bupivacaine, and lower utilization of expensive intravenous acetaminophen and intravenous buprenorphine, this protocol was associated with a marked reduction in pharmacy charges as well as overall hospital charges.

\begin{tabular}{|c|c|c|c|c|}
\hline Characteristic & Control (n=17) & ERAS (n=11) & Change & p Value \\
\hline \multicolumn{5}{|c|}{ Breakthrough Narcotic Use (mg IV Buprenorphine) } \\
\hline Day 0 & $17[0.23(0.09-0.37)]$ & $11[0.25(0.04-0.44)]$ & $+7 \%$ & $0.899^{\ddagger}$ \\
\hline Day 1 & $17[0.34(0.13-0.54)]$ & $11[0.14(0.00-0.28)]$ & $-59 \%$ & $0.16^{\ddagger}$ \\
\hline Day 2 & $17[0.35(0.12-0.59)]$ & $11[0.05(0.00-0.18)]$ & $-85 \%$ & $0.07^{t}$ \\
\hline Total by Day 2 & $17[0.92(0.45-1.40)]$ & $11[0.44(0.10-0.78)]$ & $-53 \%$ & $0.16^{\ddagger}$ \\
\hline Day 3 & $16[0.16(0.02-0.29)]$ & $7[0.02(0.04-0.09)]$ & $-83 \%$ & $0.15^{t}$ \\
\hline Total & $17[1.08(0.47-1.68)]$ & $11[0.53(0.14-0.93)]$ & $-51 \%$ & $0.18^{\ddagger}$ \\
\hline Declined IV Buprenorphine & $4(24)$ & $5(45)$ & & $0.22^{\S}$ \\
\hline Day 2 Discharge & $1(6)$ & $4(36)$ & & $0.15^{5}$ \\
\hline Needed Breakthrough Antiemetic & $5(29)$ & $1(9)$ & & $0.21^{5}$ \\
\hline \multicolumn{5}{|l|}{ Postpartum Charges (\$) } \\
\hline Pharmacy Charges & $17[2184(1922-2447)]$ & $11[1581(1345-1817)]$ & $-\$ 603$ & $0.003^{\ddagger}$ \\
\hline R\&B Charges & $17[9510(9137-9883)]$ & $11[8524(7394-9655)]$ & $-\$ 986$ & $0.04 t$ \\
\hline Total Charges & 17 [11695 (11276-12114)] & $11[10106(8883-11328)]$ & $-\$ 1589$ & $0.004^{\ddagger}$ \\
\hline \multicolumn{5}{|c|}{$\begin{array}{l}\text { Data are } \mathrm{n}[\text { mean }(95 \% \mathrm{CI})] \text { or } \mathrm{n}(\%) \\
\pm \text { Mann-Whitney U Test }\end{array}$} \\
\hline
\end{tabular}

This study examines a unique population in that $29-73 \%$ of the subjects are obese, $26-36 \%$ of patients are tobacco users, and the subjects come from an area highly addicted to opioids. This is the first study to address pain management in patients who struggle with addiction and who are on maintenance therapy. This study is among the first that evaluates the safety 
and efficacy of liposomal bupivacaine after cesarean delivery. The higher proportion of patients who use tobacco in the ERAS group may have increased the IV buprenorphine use in the ERAS group, as tobacco use is associated with higher levels of narcotic use. ${ }^{15}$ However, its relationship to buprenorphine utilization is unclear and likely did not significantly alter our results. While the intrinsic validity characteristics demonstrate the lack of randomization, a preponderance of the patient-specific characteristics that increase surgical difficulty were similar among the groups.

Our study design is a unique ambispective design containing both a retrospective, before intervention, and a prospective, after intervention, component. Although blinding was impossible due to the nature of the ERAS protocol intervention, a significant amount of attention was given to avoiding bias in the data collection process. The language used to obtain informed consent was carefully chosen to avoid artificial lowering of pain scores due to patient bias. To protect against bias from the research staff, we took care to avoid interacting with the research subjects beyond providing their necessary and routine care. To ensure accuracy of pain measurements, the nurses were instructed to record pain scores the same way for all patients. In order to avoid artificially lowering pain scores, all patients had similar quantities of opioids offered to them. Due to the heavily Caucasian population that our facility serves, we were unable to draw any meaningful conclusion regarding the effect of the ERAS protocol on minorities.

Our study is a pilot study, with a relatively small sample $(n=28)$; a larger and sufficiently powered study would require a multi-year and multi-center approach. Patients who underwent surgery earlier in the day would logically have a longer postoperative day 0 and thus use more opioids. However, this discrepancy would have a limited impact as an additional few hours of postoperative time would not account for the significant difference seen between the groups. Our study was not designed, nor intended to compare the efficacy of On- $\mathrm{Q}^{\circledR}$ and liposomal bupivacaine, rather it was designed to compare a pain management protocol that utilizes liposomal bupivacaine to a pain management protocol that utilizes $O n-Q^{\circledR}$.
Liposomal bupivacaine with an abdominal binder, scheduled oral acetaminophen and ibuprofen along with intravenous buprenorphine as needed for breakthrough pain are a safe alternative to traditional methods for patients who are undergoing treatment for opioid addiction with buprenorphine after non-emergent cesarean delivery after 34 weeks. Our protocol appears to be associated with a 27 percent reduction in pain scores and 51 percent reduction in IV buprenorphine use. This study shows the promise of liposomal bupivacaine and enhanced recovery protocols in reducing pain and opioid use postoperatively. Further prospective and larger clinical trials are warranted.

\section{AUTHOR AFFILIATIONS}

1. Marshall University Joan C. Edwards School of Medicine, Huntington, West Virginia

\section{REFERENCES}

1. Centers for Disease Control and Prevention. Injury Prevention and Control: Opioid Overdose. Drug Overdose Death Data. 2016. Accessed March 2017.

2. Opioid abuse, dependence and addiction in pregnancy. Committee Opinion No. 524.

American College of Obstetricians and Gyneoclogists. Obstet Gynecol. 2012;119;1070-6. Reaffirmed 2016.

3. Opioid Dose Calculator. [March 2017]. Agency Medical Directors Group. Available from http:// www.agencymeddirectors.wa.gov/Calculator/ DoseCalculator.htm.

4. Equivalency Table. [March 2017]. Stanford School of Medicine:Palliative Care. Available from https://palliative.stanford.edu/opioidconversion/equivalency-table.

5. Opilphant SS, Jones $\mathrm{KA}$, Want $\mathrm{L}$, Bunker $\mathrm{CH}$, Lowder JL. Trends over time with commonly performed obstetric and gynecologic inpatient procedures. Obstet Gynecol. 2010;116(5);926931.

6. Wrench IJ, Allison A, Galimberti A, Radley S, Wilson MJ. Introduction of enhanced recovery for elective caesarean section enabling next day discharge: a tertiary centre experience. 
International Journal of Obstetric Anesthesia. 2015;24:124-130.

7. Dickson EL, Stockwell E, Geller MA, Vogel RI, Mulany SA, Ghebre R, et al. Enhanced recovery program and length of stay after laparotomy on a gynecologic oncology service: a randomized control trial. Obstet Gynecol. 2017;129(2):355362.

8. Gustafsson UO, Hausel J, Thorell A, Ljungqvist $\mathrm{O}$, Soop M, Nygren J, et al. Adherence to the enhanced recovery after surgery protocol and outcomes after colorectal cancer surgery. Arch Surg. 2011;146:571-7.

9. Dickson E, Argenta PA, Reichert JA. Results of introducing a rapid recovery program for total abdominal hysterectomy. Gynecol Ovsts Invest. 2012;74:21-5.

10. Nelson G, Kalgera E, Dowdy SC. Enhanced recovery pathways in gynecologic oncology. Gynecol Oncol. 2014;135:586-94.

11. Kalogera E, Makkum-Gamez JN, Weaver AL, Moriarty JP, Borah BJ, et al. Abdominal incisions injection of liposomal bupivicain and opioid use after laparotomy for gynecologic malignancies. Obestr Gynecol. 2016;128(5):1009-1017.

12. Chapman JS, Roddy E, Ueda S, Brooks R, Chen LL, Chen LM. Enhanced recovery pathways for improving outcomes after minimally invasive gynecologic oncology surgery. Obstet Gynecol. 2016;128(1):138-44.

13. Exparel ${ }^{\circledR}$ [package insert]. Pacira Pharmaceuticals, Inc., San Diego, CA; 2016.

14. Nelson G, Dowdy SC, Lasala J, Mena G, BakkumGamez J, Meyer LA, Iniesta MD, Ramirez PT. Enhanced recovery after surgery (ERAS ${ }^{\circledR}$ ) in gynecologic oncology. Practical considerations for program development. Gynecologic Oncology. 2017;147(3)617-620.

15. Jarlenski M, Bodnar L, Kim J, Donohue J, Krans E, Bogen D. Filled prescriptions for opioids after vaginal delivery. Obstet Gynecol. 2017;129(3):431-437.

\section{ACKNOWLEDGEMENTS}

We would like to thank the nurses and staff on Labor and Delivery and Mother Baby at Cabell Huntington Hospital for their cooperation and assistance in the implementation of the protocol. 